\title{
Philosophiques
}

\section{Les Lumières et la part d'ombre de la raison pure}

\section{Charlotte Sabourin}

Volume 43, numéro 2, automne 2016

Dossier. Usages de la réflexivité en philosophie allemande

URI : https://id.erudit.org/iderudit/1038203ar

DOI : https://doi.org/10.7202/1038203ar

Aller au sommaire du numéro

Éditeur(s)

Société de philosophie du Québec

ISSN

0316-2923 (imprimé)

1492-1391 (numérique)

Découvrir la revue

Citer cet article

Sabourin, C. (2016). Les Lumières et la part d'ombre de la raison pure.

Philosophiques, 43(2), 185-206. https://doi.org/10.7202/1038203ar

\section{Résumé de l'article}

Cet article vise à souligner l'importance du rôle joué par la conception kantienne des Lumières dans la Critique de la raison pure. On verra en effet que la connaissance de soi au coeur du projet critique gagne à être comprise en relation avec l'idée de penser par soi-même. Cette connaissance de soi dévoile cependant certaines erreurs tenaces commises par la raison, qui doivent précisément être rectifiées sous peine de compromettre la liberté de penser. La conception kantienne des Lumières nous permettra ainsi de faire sens de la nature réflexive de l'entreprise critique.
Ce document est protégé par la loi sur le droit d'auteur. L'utilisation des services d'Érudit (y compris la reproduction) est assujettie à sa politique d'utilisation que vous pouvez consulter en ligne.

https://apropos.erudit.org/fr/usagers/politique-dutilisation/ 


\title{
Les Lumières et la part d'ombre de la raison pure
}

\author{
CHARLOTTE SABOURIN \\ Université McGill \\ charlotte.sabourin@mcgill.ca
}

\begin{abstract}
RÉSUMÉ. - Cet article vise à souligner l'importance du rôle joué par la conception kantienne des Lumières dans la Critique de la raison pure. On verra en effet que la connaissance de soi au cœur du projet critique gagne à être comprise en relation avec l'idée de penser par soi-même. Cette connaissance de soi dévoile cependant certaines erreurs tenaces commises par la raison, qui doivent précisément être rectifiées sous peine de compromettre la liberté de penser. La conception kantienne des Lumières nous permettra ainsi de faire sens de la nature réflexive de l'entreprise critique.
\end{abstract}

ABSTRACT. - The aim of this paper is to show that the Kantian conception of enlightenment plays an important role in the Critique of Pure Reason. I will show that the self-knowledge central to Kant's critical philosophy can be better understood by considering it in relation with the idea of thinking for oneself. Moreover, this self-knowledge reveals some deep-rooted errors made by pure reason that must be rectified in order not to compromise freedom of thought. I will thus use Kant's conception of enlightenment to make sense of the reflexive nature of the critical project.

\section{Introduction'}

Si elle a longtemps été mise à l'écart de l'entreprise critique et conséquemment discutée de façon plutôt superficielle, la conception kantienne des Lumières fait aujourd'hui l'objet d'un regain d'intérêt. Cette popularité croissante porte généralement sur le rôle des Lumières dans la philosophie pratique; de fait, dans Qu'est-ce que les Lumières? son texte le plus connu sur la question, Kant associe volontiers les Lumières à ce qu'on pourrait qualifier, suivant Fleischacker, d' " acte moral ${ }^{2}$ — celui de la sortie d'un état de minorité. De la même façon, dans l'Idée d'une histoire universelle d'un point de vue cosmopolitique, Kant évoque volontiers l'influence que doivent exercer les Lumières sur les principes de gouvernement ${ }^{3}$. On peut en retirer que les Lumières kantiennes sont indubitablement appelées à intervenir en matière de morale et de religion.

1. Au vu de l'importance attachée par Kant au fait de penser en commun, je tiens à remercier Augustin Dumont (Université de Montréal) pour sa relecture attentive et ses remarques judicieuses.

2. Fleischacker, «Kant's Enlightenment» dans What is Enlightenment? p. I6.

3. Idée d'une histoire universelle d'un point de vue cosmopolitique, Ak. VIII, 28. À l'exception de la Critique de la raison pure, les références aux œuvres de Kant sont effectuées suivant la pagination des Gesammelte Schriften (Akademie-Ausgabe) [Berlin: Walter de Gruyter, I902ff.] Les traductions utilisées sont incluses à la bibliographie. 
Nous souhaitons pour notre part insister davantage sur leur ancrage dans la philosophie théorique en soulignant le rôle qu'elles viennent jouer dans le projet même de la Critique de la raison pure. Cette association entre Lumières et critique n'est certes pas nouvelle - Michel Foucault la suggérait lui-même en 1978 dans une conférence intitulée "Qu'est-ce que la critique?", en y décrivant la critique kantienne comme "prolégomène à toute Aufklärung présente et future ${ }^{4} »$. Foucault distingue cependant critique et Lumières en décrivant la première comme «l'idée que nous nous faisons de notre connaissance et de ses limites» et en associant les secondes à une sorte d'acte de courage ${ }^{5}$. Nous espérons plutôt montrer que les Lumières possèdent une dimension éminemment théorique, d'emblée à l'œuvre dans la Critique de la raison pure, et qui vient faire sens du caractère réflexif de l'entreprise critique. Ce que nous regardons ici comme réflexivité n'est pas qualifié comme tel par Kant, mais joue néanmoins un rôle de premier plan dans la Critique, puisqu'il s'agit du nécessaire retour sur soi de la raison. Cette réflexivité fait ainsi partie intégrante du projet philosophique kantien; elle englobe à la fois la tâche de connaissance de soi que la raison doit mener à bien, et la nécessaire rectification des erreurs qu'elle commet. Les Lumières nous permettront de faire sens de cette réflexivité en l'associant au fait de penser par soi-même.

Nous procéderons pour ce faire en trois temps. Il conviendra d'abord de voir en quoi la conception kantienne des Lumières, qui ne devait être exposée qu'en $\mathrm{I} 784$, se trouve déjà préfigurée dans le projet philosophique de Kant. Ce projet repose en effet sur l'idée de penser par soi-même - et ce, tant dans la Critique de la raison pure que dans des œuvres antérieures. En effet, les doctrines philosophiques préétablies, en étant le produit d'une «raison étrangère» plutôt que de notre propre raison, ne peuvent que faire l'objet d'une connaissance "historique". Contre l'inculcation de telles doctrines, Kant insiste plutôt sur l'importance d'apprendre à philosopher. Nous proposerons ensuite, sur cette base, une mise en relation de la connaissance de soi qui fait l'objet de la Critique de la raison pure avec l'idée fondamentale de penser par soi-même. La tâche de connaissance de soi menée par la raison prend en effet la forme d'une connaissance des lois qui gouvernent son pouvoir de connaître; à ce titre, elle peut et doit être comprise par rapport à la liberté de penser caractéristique des Lumières kantiennes. L'opuscule Qu'est-ce que s'orienter dans la pensée? fournira d'utiles précisions à ce sujet, car Kant y définit la liberté de penser non plus uniquement par rapport à des enjeux pratiques comme c'était le cas dans Qu'est-ce que les Lumières? mais bien comme le fait de chercher en soi-même la pierre de touche de la vérité et en soulignant l'importance, à cet égard, des lois régissant notre pouvoir de connaître. Car la liberté de penser sans lois, sur le plan

4. Foucault, "Qu'est-ce que la critique?", p. 4I.

5. Ibid, p. 4I. 
spéculatif, est celle du « génie » — qui valse dans la Schwärmerei et la superstition. Enfin, on verra que les Lumières possèdent une dimension négative qui se révèle fort utile à la rectification de certaines erreurs tenaces évoquées dans la Critique de la raison pure. Ces erreurs, et tout particulièrement celle que cause l'inévitable apparence transcendantale, doivent être prises en considération dans le retour sur soi qui caractérise les Lumières à l'œuvre dans la Critique; car, bien loin d'être instantanée, cette réflexivité, en ce qu'elle se heurte à un obstacle persistant à la source de l'erreur, doit être comprise comme une entreprise toujours réitérée.

\section{Les Lumières kantiennes avant 1784. Une conception éclairante de la philosophie}

Une première observation s'impose: comme on le sait, la conception kantienne des Lumières n'a été exposée en bonne et due forme qu'en I 784 , avec la parution de "Qu'est-ce que les Lumières?». On peut donc se demander à quel point il est légitime d'espérer la voir déjà à l'œuvre dans la première édition de la Critique de la raison pure. Or Kant se servait déjà du terme d'Aufklärung (ainsi que du verbe correspondant, aufklären) en I78 I mais en un sens quelque peu différent de celui qu'il devait revêtir à compter de $\mathrm{I}_{784}$. Si Kant fait déjà, dès la première édition de la Critique, quelques remarques sur l'avènement prochain des Lumières (par exemple, lorsqu'il prédit, dans sa préface, «une prochaine transformation » et «une prochaine avancée » des Lumières dans les sciences $\left.{ }^{6}\right)$, la plupart de ses remarques en la matière se rapportent plutôt à une "clarification" (ou éclaircissement) de concepts qu'au mouvement des Lumières proprement dit $^{7}$. Cette entreprise de clarification est d'ailleurs au cœur d'un passage de la «Discipline de la raison pure » où Kant nous rappelle que la raison, en philosophie, doit éviter d'emprunter les méthodes de la mathématique:

Ce sont là de vaines prétentions qui jamais ne peuvent aboutir, mais qui bien plutôt doivent faire revenir la philosophie à son dessein de découvrir les illusions d'une raison méconnaissant ses limites et ramener, par l'intermédiaire

6. Critique de la raison pure (ci-après: $C R P), A X$. Les références à la Critique de la raison pure sont effectuées suivant la pagination standard $\mathrm{A} / \mathrm{B}$ correspondant aux éditions de I78I et I787.

7. Voir à ce sujet l'étude approfondie de Kreimendahl, «Kants vorkritisches Programm der Aufklärung». Il y expose en détail les racines de la notion d'Aufklärung entendue comme clarification conceptuelle, en soulignant qu'elle n'avait pas encore acquis la dimension émancipatrice qui caractérisera les Lumières kantiennes proprement dites. Ce qui doit être éclairé est, en ce cas, moins l'humanité ou le siècle que la raison elle-même - et cet "éclairement" est surtout à comprendre comme entreprise épistémologique. L'analyse de Kreimendahl est ellemême fort éclairante; mais il nous semble malgré tout que cette clarification conceptuelle puisse s'insérer dans une conception des Lumières déjà à l'œuvre dans la première Critique, puisqu'elle est fondée, comme on le verra, sur l'idée de se fier à sa propre raison plutôt qu'à une autorité extérieure. 
d'une clarification [Aufklärung] suffisante de nos concepts, la présomption de la spéculation à une connaissance de soi-même modeste, mais solidement étayée ${ }^{8}$.

Quoique Kant ne définisse pas cette clarification conceptuelle dans la Critique, on peut considérer qu'elle englobe non seulement la détermination des limites de l'application des concepts, obtenue grâce à l'exposition des concepts d'espace et de temps et à la déduction des concepts purs de l'entendement, mais aussi une réflexion transcendantale sur ces mêmes concepts - laquelle consiste à en déterminer le lieu, c'est-à-dire la source dont ils sont issus (sensibilité ou entendement). Ainsi, quoique l'Aufklärung entendue comme clarification conceptuelle ne soit évidemment pas équivalente à l'Aufklärung de I784, elle s'inscrit d'emblée dans l'esprit de l'entreprise critique9. Le passage de la "Discipline" cité plus haut montre par ailleurs que la clarification conceptuelle participe d'une nécessaire connaissance de soimême - laquelle doit être effectuée par la raison elle-même et non par une autorité extérieure, comme c'est le cas des philosophies non critiques. C'est d'ailleurs ce que met en évidence un passage subséquent de la Discipline:

[II] y a quelque chose de fortement absurde dans le fait d'attendre de la raison un éclairage [Aufklärung] et de lui prescrire pourtant à l'avance de quel côté il lui faut nécessairement se diriger ${ }^{10}$.

De fait, c'est par la critique que la raison doit devenir «éclairée » [aufgeklärte] (cf. $A_{755} / B_{7} 83$ ), puisque c'est par elle que la raison procède à l'examen de son propre pouvoir de connaître et, ce faisant, remonte à la source de ses jugements et de ses concepts. Quoiqu'on gagne à distinguer l'Aufklärung en tant que clarification conceptuelle de la conception plus achevée des Lumières, elle semble bel et bien préfigurer l'idée de penser par soi-même, qui, comme on le verra à présent, est constitutive non seulement du projet des Lumières, mais aussi de la conception kantienne de la philosophie.

En effet, bien qu'elle vienne mobiliser cette caractérisation de l'Aufklärung comme clarification, la Critique de la raison pure nous semble s'inscrire tout de même dans une conception générale de la philosophie reposant sur un thème fondamental des Lumières kantiennes — soit l'idée de penser par soi-même. De fait, Kant n'a pas attendu I 784 pour mettre de

8. CRP, $\mathrm{A}_{735} / \mathrm{B}_{7} 63$. Voir aussi, parmi les occurrences les plus significatives de Aufklärunglaufklären: A537/B565; A747/B $775 ; \mathrm{A}_{755} / \mathrm{B}_{783}$; A848/B876.

9. À ce sujet, on se rapportera avec profit à l'article "La philosophie kantienne de l'histoire et la ruse des Lumières » de C. Piché, qui souligne, suivant Timmermann, la présence du participe passé aufgeklärt dans la Fondation de la métaphysique des mœurs à propos du concept de volonté bonne, lequel nécessite «moins d'être inculqué que simplement éclairci » (cf. Ak. IV, 397).

10. $C R P, \mathrm{~A}_{747} / \mathrm{B}_{775}$. Nous suivons ici la traduction de Renaut. Soulignons cependant que Tremesaygues et Pacaud traduisent ici Aufklärung par «des lumières ", tout comme, d'ailleurs, Guyer et Wood («enlightenment»). 
l'avant une telle vision de la philosophie, déjà évoquée dans un passage de la Critique devenu célèbre:

On ne peut donc, parmi toutes les sciences rationnelles (a priori) apprendre seulement que la mathématique, mais jamais la philosophie (si ce n'est historiquement): en fait, pour ce qui concerne la raison, on ne peut apprendre tout au plus qu'à philosopher $(\mathrm{A} 837 / \mathrm{B} 865)^{11}$.

En effet, plutôt que l'apprentissage de doctrines, c'est un exercice de la raison bien particulier qui doit, pour Kant, caractériser la philosophie. Cet exercice vise certes, dans la Critique, à paver la voie à une philosophie entendue comme système (c'est-à-dire: comme unité des diverses connaissances sous une idée ${ }^{12}$ ) - un système caractérisé par la mise en œuvre des principes universels de la raison, qui sont, on s'en doute bien, ceux mis au jour par la Critique de la raison pure. Mais la philosophie englobe également une propédeutique indissociable du système: l'entreprise critique ellemême. Celle-ci consiste en un examen, mené par la raison elle-même, de ses propres principes quant à leurs sources, afin d'attester de leur validité. C'est là, on s'en doute, une contribution majeure de la Critique de la raison pure - ainsi que, comme nous l'apprend l'Architectonique, un droit qui doit toujours être réservé à la raison ${ }^{13}$. Ainsi, la philosophie englobe bel et bien une doctrine, si on entend par là le système achevé constitué par la mise en œuvre des principes universels de la raison - mais cette doctrine doit toujours être accompagnée d'une critique, c'est-à-dire d'un examen adéquat, effectué par la raison, de son propre pouvoir de connaître ${ }^{14}$.

Ce que Kant reproche aux doctrines de ses prédécesseurs est précisément d'avoir voulu se dispenser d'une telle critique - qui aurait pourtant montré l'inexactitude des principes sur lesquels elles sont fondées. C'est en ce sens que le système de Wolff, par exemple, ne peut faire l'objet que d'une connaissance «historique » : du point de vue de celui qui l'examine, il s'est toujours constitué «d'après une raison étrangère ${ }^{15}$ ». Tout dogmatisme propose ainsi une connaissance pure par concepts, mais sans investiguer la source de ces principes ou la façon dont la raison les aurait obtenus: bref, en croyant pouvoir se passer de cette propédeutique que constitue l'examen du pouvoir de connaître lui-même. Le dogmatique se repose ce faisant non sur

11. Voir aussi la même remarque dans l'Annonce du programme des leçons de M. E. Kant durant le semestre d'hiver (I765-I766) (ci-après: Annonce): "L'enfant, au terme de sa scolarité, était habitué à apprendre. Il pense maintenant qu'il va apprendre la philosophie, mais c'est impossible, car il doit désormais apprendre à philosopher" (Ak. II, 306).

12. $\mathrm{CRP}, \mathrm{A} 832 / \mathrm{B} 860$; voir aussi à ce sujet $\mathrm{B} 27$ sq.

13. $\mathrm{CRP}, \mathrm{A} 838 / \mathrm{B} 866$.

14. Par ailleurs, Piché montre bien, contre Lyotard, que, bien que Kant distingue soigneusement doctrine et critique, les deux notions ne sont pas radicalement opposées: de fait, la première Critique en contient une, la Doctrine transcendantale de la faculté de juger (ou analytique des principes) («The Philosopher-Artist: A Note on Lyotard's Reading of Kant », p. I 55 ).

15. CRP, A836/B864; l'italique est de moi. 
sa propre raison, mais bien sur une raison étrangère; alors que c'est en notre propre raison qu'il convient de chercher la pierre de touche de la vérité - ce qui est, on le verra, une idée fondamentale de la conception kantienne des Lumières. D'où la participation de la clarification conceptuelle évoquée plus haut à cette conception de la philosophie. Cette insistance sur l'usage de notre propre raison plutôt que sur l'apprentissage de doctrines historiques a en outre de fortes répercussions sur le plan pédagogique, puisqu'elle suggère que l'élève doit être amené graduellement à penser par lui-même.

Enfin, il est intéressant de constater que l'importance d'apprendre non pas la philosophie, mais bien à philosopher était déjà soulignée par Kant bien avant la Critique, dans l'annonce du programme de ses leçons du semestre d'hiver I765-I766. Il y insiste en effet sur l'importance de former l'entendement de l'élève et de guider ce dernier pour qu'il parvienne à philosopher par lui-même plutôt que de lui inculquer des doctrines préétablies. C'est précisément parce qu'on a négligé cette formation essentielle de l'entendement que, nous dit-il,

il n'est pas rare de rencontrer des savants (à proprement parler des gens qui ont fait des études) qui montrent peu d'entendement, et c'est pourquoi les académies envoient par le monde plus d'esprits insipides que n'importe quel autre corps social ${ }^{16}$.

Kant s'y montre tout aussi sévère vis-à-vis de la tradition philosophique, qui ne doit être utilisée, précise-t-il, que dans le but d'apprendre à l'élève à porter lui-même un jugement sur les auteurs à l'étude. Ce qui doit être au centre de l'enseignement de la philosophie est bien plutôt «la méthode pour réfléchir et décider par soi-même ${ }^{17}$ ".

L'importance rattachée au fait de penser par soi-même n'est donc pas uniquement exploitée dans les textes consacrés spécifiquement aux Lumières, puisqu'il s'agit d'une idée que Kant rattache à sa conception même de la philosophie - et bien avant I784. Cette association est constante: on peut la remarquer jusque dans son cours de logique, alors qu'il précise qu' "on n'apprend à philosopher que par l'exercice et par l'usage qu'on fait soimême de sa propre raison ${ }^{18}$ ». La nécessité de penser par soi-même est ainsi pour lui constitutive de la philosophie. Notons au passage que Kant est tout à fait conscient de la difficulté inhérente à cette entreprise: penser par soimême n'est pas un exercice naturel ou quelque chose qui irait de soi, au contraire - l'entendement doit y avoir été adéquatement préparé. L'importance pour les Lumières d'exercer l'entendement à cet égard sera d'ailleurs soulignée dans Qu'est-ce que les Lumières?19, un sujet sur lequel nous aurons l'occasion de revenir.

16. Annonce, Ak. II, 306.

17. Annonce, Ak. II, 307.

18. Logique Jäsche, Ak. IX, 25-26.

19. Qu'est-ce que les Lumières? (ci-après: QL), Ak. VIII, 36. 


\section{Penser par soi-même et se connaître soi-même.}

La Critique peut ainsi d'emblée être comprise sur la base d'un projet philosophique anticipant certains aspects de la pensée des Lumières telle qu'elle sera exposée à compter de I 784 - sans bien sûr cesser d'évoluer. Nous aimerions à présent montrer que cette pensée des Lumières permet également de thématiser le caractère réflexif de l'entreprise critique, laquelle vient mobiliser une nécessaire connaissance de soi de la raison. Cette connaissance de soi est, comme on le verra, intrinsèquement reliée au fait de penser par soi-même.

Le texte kantien le plus célèbre portant sur les Lumières est et restera sans doute Qu'est-ce que les Lumières? - pour des raisons évidentes. Kant y livre l'essentiel de sa conception de l'Aufklärung, qui, comme le confirmera son intervention subséquente dans la querelle du panthéisme, diffère grandement de celles de ses contemporains, Mendelssohn au premier $\operatorname{chef}^{20}$. Or les thèmes abordés dans l'opuscule de I 784 concernent en grande partie la mise en œuvre des Lumières dans un contexte social et politique, ce qui peut donner l'impression que la conception kantienne des Lumières est intrinsèquement pratique — ou, du moins, limitée à l'usage pratique de la raison. En effet, dès le premier paragraphe du texte, Kant situe résolument les Lumières dans un contexte pratique et social - ce qui, doit-on le souligner, est peu habituel chez l'auteur des trois Critiques:

Les Lumières se définissent comme la sortie de l'homme hors de l'état de minorité, où il se maintient par sa propre faute. La minorité est l'incapacité de se servir de son entendement sans être dirigé par un autre. Elle est due à notre propre faute quand elle résulte non pas d'un manque d'entendement, mais d'un manque de résolution et de courage pour s'en servir sans être dirigé par un autre. Sapere aude! Aie le courage de te servir de ton propre entendement! Voilà la devise des Lumières ${ }^{21}$.

Par ailleurs, tout en soulignant la responsabilité de chacun vis-à-vis son propre état de minorité (puisque c'est par «paresse» et par «lâcheté » qu'on y demeure confiné), il faut noter que Kant insiste tout particulièrement, dans cet opuscule, sur ce que Qu'est-ce que s'orienter dans la pensée? qualifiera $d^{\prime}$ ' «obstacles extérieurs » pouvant entraver la progression des Lumières ${ }^{22}$. En

20. Zöller prend cependant soin de souligner que la position de Kant ne rompt pas complètement avec celle de Mendelssohn - tous deux associant les Lumières au progrès de la connaissance ainsi qu'à la destination de l'humanité («Aufklärung über Aufklärung. Kants Konzeption des selbständigen, öffentlichen und gemeinschaftlichen Gebrauchs der Vernunft », p. 87).

21. QL, Ak. VIII, 36 ; trad. modifiée.

22. Qu'est-ce que s'orienter dans la pensée? (ci-après: QO), Ak. VIII, I47. Le résultat peut apparaitre un peu paradoxal: ainsi, pour Kant, chacun est responsable de son propre état de minorité, tout en y étant parfois maintenu de force par un tuteur bienveillant. Le cas des femmes est particulièrement intéressant à cet égard: Kant prend la peine de mentionner dans 
effet, Qu'est-ce que les Lumières? est consacré plus particulièrement à l'étude du rapport entre Lumières et liberté civile: «Or, pour répandre ces lumières, il n'est rien requis d'autre que la liberté; et à vrai dire la plus inoffensive de toutes les manifestations qui peuvent porter ce nom, à savoir celle de faire un usage public de sa raison dans tous les domaines ${ }^{23}$." La distinction bien connue entre usage public et usage privé de la raison vient en effet mobiliser une conception particulière de la liberté d'expression: Kant la juge nécessaire à la diffusion des Lumières, mais seulement en ce qui concerne l'usage public de la raison - c'est-à-dire, celui qu'on en fait «en tant que savant, devant l'ensemble du public qui lit ${ }^{24}$ ». L'usage privé de la raison, effectué dans le contexte strict d'une charge civile ou de fonctions professionnelles, peut quant à lui demeurer limitées .

De fait, la liberté civile ou liberté d'expression est si étroitement liée au projet des Lumières qu'on gagne sans doute à comprendre celles-ci dans un contexte social et politique. Mais cela peut donner l'impression que tout se joue sur le plan pratique, alors que, comme le montrera Qu'est-ce que

Qu'est-ce que les Lumières? que la plupart des êtres humains, incluant toutes les femmes, sont en état de minorité (Ak. VIII, 36) - sans plus d'explication. Cet exemple de Kant pourrait être compréhensible s'il constituait une simple constatation portant sur le statut social des femmes, mais son insistance sur la responsabilité de chacun (et chacune) dans son propre état de tutelle rend difficile d'en faire une interprétation charitable. J. G. Hamann reviendra précisément sur cet exemple pour illustrer le paradoxe de la position kantienne dans une lettre à C. J. Kraus (I 8 décembre I784), alléguant que cette idée d'un état de tutelle dans lequel toutes les femmes se maintiendraient d'elles-mêmes n'est pas près d'être acceptée de ses trois filles!

23. QL, Ak. VIII, 36 .

24. QL, Ak. VIII, 37. Kant insistait d'ailleurs déjà sur ce libre usage public de la raison dans la Discipline de la raison pure, dans un passage abondamment discuté: "À cette liberté appartient donc aussi celle d'exposer publiquement au jugement ses pensées et les doutes que l'on ne peut réduire soi-même, sans être pour autant décrié comme un citoyen agité et dangereux. C'est là un point qui se trouve déjà compris dans le droit originaire de la raison humaine, laquelle ne connaît pas d'autre juge qu'à nouveau l'universelle raison humaine, où chacun a sa voix; et dans la mesure où c'est de cette dernière que doivent provenir toutes les améliorations dont notre état est susceptible, un tel droit est sacré et il ne peut y être attenté» (CRP, $\mathrm{A}_{75}$ 2/B 780 ).

25. Kant justifie cette limitation de l'usage privé de la raison en expliquant que certains membres d'une communauté doivent, dans le cadre de leurs fonctions, se laisser diriger passivement en vue de l'intérêt du groupe. Cela implique qu'ils se soumettent alors à une autorité extérieure (celle de leur supérieur) plutôt qu'à l'autorité de leur propre raison - d'où, selon O'Neill, le choix du terme "privé»: "Il s'agit d'usages privés [“deprived"] (privatus), car incomplets, de la raison. Il y a dans de tels rapports une prémisse tacite, injustifiée et non critiquée de soumission face à l'«autorité " conférée par une fonction. L'antithèse de ces usages privés et partiels de la raison doit être un usage public plus complet de la raison, qui refuse fermement cette dépendance envers des autorités puissantes, mais infondées, au profit d'une autodiscipline" (Constructions of Reason: Explorations of Kant's Practical Philosophy, p. I7; ma traduction). Kant laisse malgré tout en suspens, du moins dans ce texte, la question de savoir si cette limitation de l'usage privé de la raison est nécessaire aux Lumières - il précise, en Ak. VIII, 37, que la limitation de l'usage privé de la raison est favorable aux Lumières, mais rajoute quelques lignes plus bas que cet usage privé "peut souvent être très étroitement limité sans pour autant entraver notablement le progrès des Lumières". 
s'orienter dans la pensée? s'il est vrai que la contrainte civile nuit à la liberté de penser, elle est pourtant loin d'en constituer la seule menace. Cette constatation doit cependant être comprise dans le contexte plus large des circonstances ayant mené à la parution de l'opuscule. On se souvient qu'il constitue en effet l'unique intervention publique de Kant dans la célèbre querelle du panthéisme opposant, au premier chef, Mendelssohn et Jacobi - mais impliquant nombre d'autres philosophes au passage, comme, par exemple, Wizenmann, dont Kant évoque les Résultats de la philosophie de Jacobi et de Mendelssohn. Kant ne se résoudra à intervenir dans la querelle qu'en octobre I786 avec la publication, dans la Berlinische Monatsschrift, de Qu'est-ce que s'orienter dans la pensée? Désirant tout à la fois défendre la Critique de la raison pure contre les accusations de spinozisme portées par Jacobi et communiquer sa propre perspective sur la querelle et sur les Lumières, Kant doit s'expliquer non seulement avec Mendelssohn, dont les vues sont déjà mises à mal par les critiques de Jacobi et de Wizenmann, mais aussi avec Jacobi et le concept de Schwärmerei (exaltation de l'esprit ${ }^{26}$ ) que Kant lui prête - tout en l'associant non à un irrationalisme proprement dit, mais bien à une raison qui ne reconnaîtrait plus aucune loi. Kant articule ce concept de la façon suivante:

Nous autres, en hommes du commun, nous appelons «exaltation de l'esprit» [Schwärmerei] la maxime dès lors admise de l'invalidité d'une raison souverainement législatrice; mais ces favoris de la bonne nature [la nomment]: «illumination» $[\text { Erleuchtung }]^{27}$.

C'est sur cette base que la troisième section du texte de Kant vient s'opposer à la figure du "génie » de Jacobi, qui verse inévitablement, pour lui, dans cette exaltation de l'esprit. Kant y fait valoir que le génie, à force de rejeter l'idée d'une raison régie par des lois (quelles qu'elles soient) au nom de la liberté de penser, en viendra malgré lui à anéantir cette dernière en asservissant la raison aux faits. Le génie veut en effet aller au-delà des limites prescrites par la raison elle-même, ce qui le pousse à les outrepasser et à les déclarer nulles et non avenues - et, ce qui est plus grave encore, il en encourage d'autres, par ses belles paroles, à suivre son exemple. Or, pour Kant, la raison est ainsi faite qu'elle ne peut supporter très longtemps un tel état de non-loi : ceux qui cherchent à l'y maintenir, pour peu qu'ils voudront s'accorder entre eux, seront forcés d'avoir recours à une nouvelle forme de légalité, celle que permettent les faits, sombrant ainsi dans la superstition.

Le petit texte de Kant ne se veut évidemment pas une exposition détaillée des positions de ses adversaires, mais propose plutôt une interprétation (assez libre) du conflit et des dérives auxquelles on peut s'attendre de part et d'autre. Cette interprétation permet néanmoins de mieux circons-

26. Nous suivons ici la traduction de Renaut.

27. QO, Ak. VIII, I45; trad. modifiée. 
crire la liberté de penser telle que l'entend Kant. Car dans la troisième section de son opuscule, Kant s'oppose à Jacobi précisément sur le terrain de la liberté de penser, en la définissant par contraste avec trois états: i) celui de la contrainte civile, sur laquelle, on l'a vu, Qu'est-ce que les Lumières? insistait davantage; ii) celui de la contrainte exercée sur la conscience par des tuteurs, tout particulièrement en matière de religion - un aspect également très important dans Qu'est-ce que les Lumières?; et iii) la menace que représente une raison sans lois, que Kant contraste avec l'état d'une raison qui s'impose à elle-même de nécessaires restrictions dans le domaine de la pensée $^{28}$. Sur ce point, on l'a vu, Kant et le "génie» (ou, plus exactement: Kant et l'interprétation kantienne du génie) ne peuvent s'accorder. Mais ces développements rajoutent une dimension substantielle à la liberté de penser caractéristique des Lumières kantiennes, qui doit ainsi être comprise comme toujours régie par des lois: les lois de la raison elle-même. La liberté de penser est en effet menacée non seulement par toute contrainte civile limitant l'usage public de la raison et par l'état de minorité intellectuelle dont profitent les tuteurs, mais aussi par tout usage de la raison qui outrepasse ses propres lois. C'est sur cette base que Kant introduit un aspect supplémentaire de sa conception des Lumières dans Qu'est-ce que s'orienter dans la pensée? dans une note qu'il vaut la peine de citer dans son intégralité:

Penser par soi-même signifie: chercher la pierre de touche de la vérité en soi - c'est-à-dire en sa propre raison. Et la maxime de toujours penser par soimême est l'Aufklärung. Cela suppose bien moins que ne se l'imaginent ceux qui veulent voir l'Aufklärung dans les connaissances acquises; elle consiste plutôt en un principe négatif de l'usage de la faculté de connaître et il arrive souvent que celui qui est abondamment pourvu de connaissances soit le moins éclairé sur leur usage ${ }^{29}$.

Qu'est-ce que s'orienter dans la pensée? vient ainsi confirmer que les Lumières ont un rôle à jouer non pas seulement dans l'usage pratique de la

28. La symétrie est ici rompue, car Kant ne parle plus, dans ce troisième point, de contrainte s'opposant à la liberté de penser. De fait, on peut remarquer que le raisonnement semble ici renversé, puisqu'il suggère que la liberté de penser doit comporter en elle-même une forme de contrainte: celle des restrictions que s'impose la raison elle-même par l'entremise des lois régissant son pouvoir de connaître. C'est cependant la raison qui s'impose cette contrainte à elle-même, par contraste avec la contrainte imposée de l'extérieur qui guette immanquablement une raison sans lois et qui la pousse vers l'asservissement aux faits. Cette forme de contrainte auto-imposée sur le plan théorique ne va pas sans rappeler la contrainte liée à la loi morale dans la sphère pratique, mais il convient, malgré tout, de distinguer les deux formes de législation à l'œuvre. Seule la seconde est une autolégislation proprement dite; les lois régissant l'usage théorique de la raison pure sont quant à elles des lois que la raison "trouve" ou découvre en elle. Au sujet de cette distinction complexe, il vaut la peine de prendre connaissance de l'analogie proposée par O. Höffe entre raison pure et républicanisme ( La raison kantienne est-elle républicaine? Essai de lecture politique de la Critique de la raison pure»).

29. QO, Ak. VIII, I47n. 
raison, mais également en ce qui a trait à son usage théorique. Chercher la pierre de touche de la vérité en soi $^{30}$, c'est ni plus ni moins le projet de la Critique de la raison pure et ce pourquoi la raison y procède à un examen de son propre pouvoir de connaître. Cette importante dimension réflexive de l'entreprise critique prendra la forme, comme le suggérait le passage de la Discipline cité plus haut, d'une "connaissance de soi-même modeste, mais solidement étayée ${ }^{31}$ ", devant prendre la place des vaines prétentions à la spéculation. Or, cette connaissance de soi [Selbsterkenntnis] n'est pas aisée à accomplir: c'est en fait la tâche "la plus difficile de toutes" évoquée dans la première préface ${ }^{32}$ - Kant, en I796, la qualifiera même de "travail herculéen $^{33}$ ". Il faut noter que cette connaissance de soi n'est pas à entendre comme une connaissance de soi subjective ou individuelle - et en aucun cas comme l'aspiration à une connaissance de soi par la conscience intérieure que nous avons de nous-mêmes. On sait qu'il s'agit là, pour Kant, d'une dérive de la psychologie rationnelle, qui croit pouvoir élargir la connaissance que nous avons de nous-mêmes en prenant l'unité de l'aperception du sujet pour une intuition du sujet lui-même, en tant que substance. Or, l'unité de l'aperception ne fournit précisément pas d'intuition du sujet: d'où le fait que la catégorie de la substance ne puisse s'y appliquer ${ }^{34}$. La connaissance de soi qui nous intéresse ici est plutôt à la fois celle de tout être raisonnable et d'aucun en particulier : il s'agit, à proprement parler, du «soi » de la raison elle-même ${ }^{35}$. De fait, la connaissance de soi dont est capable la raison doit passer par un processus de légitimation, car les critères qu'elle met au jour doivent valoir et pouvoir être reconnus par tout être raisonnable.

C'est précisément là, nous semble-t-il, le sens qu'il faut donner à certaines remarques de prime abord un peu étonnantes de Kant, comme par exemple celle de l'idée d'un débat «à mener avec soi-même » évoquée à la fin

30. La Réflexion n $\mathrm{n}^{\circ}$ 6204, dont Adickes situe la rédaction entre les années I780-1789, propose une remarque similaire: «Être éclairé signifie: penser par soi-même, chercher en soimême la pierre de touche [suprême] de la vérité [de mon jugement. Le fondement de l'assentiment; car je dois en répondre.] C'est-à-dire dans les principes.» (Ak. XVIII, 488; trad. S. Grapotte) Kant y souligne par ailleurs que la Schwärmerei consiste à rechercher la pierre de touche de la vérité dans les sensations de l'esprit, tandis que la superstition la recherche dans les faits.

31. $C R P, \mathrm{~A}_{735} / \mathrm{B} 763$.

32. $C R P, A X I$.

33. Dans un très beau passage de son opuscule Sur un ton supérieur nouvellement pris en philosophie: «[...] mais à nul autre qu'au philosophe de l'intuition, qui effectue ses démonstrations non pas grâce au travail herculéen de la connaissance de soi, de bas en haut, mais, franchissant d'un bond ce travail, de haut en bas, grâce à une apothéose qui ne lui coûte rien, à nul autre il ne peut venir à l'esprit d'affirmer sa supériorité: car il parle là sous sa propre autorité, et c'est pourquoi il ne doit en rendre compte à personne» (Ak. VIII, 390).

34. Voir notamment en CRP, $\mathrm{A}_{3} 66$ et $\mathrm{B}_{42}$ I.

35. La personnification de la raison est un procédé courant dans l'œuvre de Kant: c'est d'ailleurs sa "vanité de beaucoup savoir" qui est à l'origine de l'entreprise critique (CRP, $\left.\mathrm{B}_{2} \mathrm{I}\right)$. 
de Qu'est-ce que s'orienter dans la pensée? - débat visant à établir s'il est "possible de prendre pour un principe universel de l'usage de la raison celui en vertu duquel on admet quelque chose ou bien encore la règle qui dérive de ce que l'on admet ${ }^{36} »$. Cette image va d'ailleurs tout à fait dans le sens d'une remarque bien connue de la Critique de la raison pure insistant sur le fait que rien ne peut se soustraire à la critique, car «la raison n'a pas d'autorité dictatoriale, mais ne fait jamais reposer sa décision que sur l'accord de libres citoyens, dont chacun doit pouvoir exprimer ses objections, voire son veto, sans retenue aucune ${ }^{37}$ ». Cette remarque ne doit bien sûr pas être reçue en un sens littéral: l'autorité de la raison et de ses principes ne repose pas sur une sorte de délibération démocratique qui permettrait de retenir comme principe de la raison tout ce qui ferait consensus. Au contraire, il ne fait aucun doute que Kant adhère à une conception classique de la vérité entendue comme accord entre une connaissance et son objet ${ }^{38}$. L'une des retombées positives de la logique transcendantale est précisément d'avoir montré que les principes de l'entendement, en rendant possible à la fois l'expérience et les objets de l'expérience ${ }^{39}$, sont non seulement vrais a priori, mais encore «la source de toute véritét0 ${ }^{»}$ - puisqu'en rendant possibles les objets de l'expérience, ils rendent du même coup possible l'accord de notre connaissance avec ces objets. C'est donc, en ce sens, les concepts purs de l'entendement qui constituent la référence avec laquelle les objets doivent s'accorder, et non l'inverse.

Et de fait, la légitimité des principes de la raison doit être attestée par des procédures en bonne et due forme, comme le font les déductions de la Critique de la raison pure. Mais leur autorité devrait pouvoir être constatée par tout être raisonnable, du moins en principe ${ }^{41}$. On l'a vu, Kant n'insinue pas que la critique soit le fruit d'un consensus: il nous rappelle plutôt qu'il existe une pluralité d'êtres raisonnables et que ceux-ci, pour peu qu'ils soient en mesure de se soumettre librement à la critique, devraient pouvoir arriver aux mêmes résultats ${ }^{42}$ - par opposition, par exemple, à une doctrine philo-

36. QO, Ak. VIII, I47n.

37. $\mathrm{CRP}, \mathrm{A} 739 / \mathrm{B} 767$.

38. Voir notamment en A58/B82: «La définition nominale de la vérité, selon laquelle elle consiste dans la conformité de la connaissance avec son objet, est ici accordée et présupposée».

39. Car « les conditions de la possibilité de l'expérience en général sont en même temps conditions de la possibilité des objets de l'expérience» (AI 5 8/BI97).

40. CRP, $\mathrm{A}_{23} 37 \mathrm{~B} 286$.

41. Rappelons tout de même que la Critique de la raison pure n'est pas une œuvre vouée à être populaire, non seulement à cause de la grande complexité des enjeux qu'elle aborde, mais également parce que les questions qu'elle soulève sont peu susceptibles d'intéresser le grand public (qui «n'entend absolument rien à de si subtils travaux»); elle s'adresse avant tout aux académiciens, lesquels sont les plus à risque de spéculer à tort et à travers sur tout ce qui touche au domaine de la raison pure (A746/B774; voir aussi AXVIII et BXXXIV).

42. C'est dans une perspective similaire que R. Meerbote redéfinit, dans «Kant's Use of the Notions "Objective Reality" and "Objective Validity" ", la notion de validité objective en 
sophique dont la raison étrangère fait autorité (comme le suggérait le passage en $C R P, \mathrm{~A} 836 / \mathrm{B} 864$ discuté plus haut ${ }^{43}$. Ce qui vient d'ailleurs mobiliser le sens commun tel que le présente la troisième Critique, c'est-à-dire cette faculté qu'ont les êtres humains de tenir compte du mode de représentation d'autrui, "pour en quelque sorte comparer [leur] jugement à la raison humaine tout entière ${ }^{44} »$. La raison ne peut être réellement universelle que si chacun y "a sa voix» et peut "exposer publiquement au jugement ses pensées et ses doutes ${ }^{45} »$. La Critique de la raison pure respecte en ce sens l'exigence de publicité posée par Qu'est-ce que les Lumières? La première préface soulignait déjà, dans une note bien connue, l'importance de ne rien soustraire au «libre et public examen" de la raison ${ }^{46}$. Cette exigence de publicité implique que la raison ne se soumette qu'à sa propre autorité, et non à l'autorité extérieure caractérisant les usages simplement privés de la raison ${ }^{47}$.

C'est sur la base de telles indications et dans la perspective des Lumières kantiennes que certaines conceptions de la raison comme «collective» ou «pluraliste» ont été proposées. Onora O’Neill conçoit ainsi l'autorité de la raison pure comme une "tâche collective ", semblable à celle d'une autorité politique (Constructions of Reason: Explorations of Kant's Practical Philosophy, p. I 8 sq.); et Allan Wood, contre la lecture habermassienne, évoque une raison kantienne pluraliste, qui nécessiterait interaction et communication avec autrui pour se développer et parvenir à maturité (Kant's Ethical Thought, p. 30I sq.) Ces deux interprétations suggèrent ainsi une raison pure fortement inspirée du modèle des Lumières, comprises comme une forme d'éducation publique et collective ${ }^{48}$. De fait, malgré le caractère individualiste de l'injonction à penser par soi-même, Kant souligne à maintes reprises le caractère collectif des Lumières, comme par exemple lorsqu'il remarque qu'il est plus facile pour un public d'accéder aux Lumières que pour un individu isolé, et ce, en raison précisément de la présence de quelques

la fondant sur un critère de communicabilité, permettant ainsi de tenir compte des développements de A820/B848 - A824/B852.

43. Au sujet du parallèle maintes fois constaté entre raison spéculative et politique chez

Kant, voir notamment O'Neill (Constructions of Reason: Explorations of Kant's Practical Philosophy) et Höffe ( «La raison kantienne est-elle républicaine? Essai de lecture politique de la Critique de la raison pure»).

44. Critique de la faculté de juger (ci-après: CFJ) Ak. V, 293.

45. $C R P, \mathrm{~A}_{75} 2 / \mathrm{B} 780$.

46. CRP, AXIn.

47. Voir O'Neill (Constructions of Reason: Explorations of Kant's Practical Philosophy, p. I7). Sur l'usage que fait Kant des notions de public et de publicité, on consultera avec profit les études exhaustives de Davis ("Kant's Different "Publics" and the Justice of Publicity») et Laursen ( "The Subversive Kant: The Vocabulary of "Public" and "Publicity" »).

48. Sur la conception kantienne de l'éducation dans le contexte du projet général des Lumières, on peut aussi consulter l'étude de R. Louden («Education» dans The World We Want: How and Why the Ideals of the Enlightenment Still Elude Us). 
individus éclairés au sein d'une communauté, qui peuvent, de par leur exemple, en encourager d'autres à sortir de leur état de minorité. Par ailleurs, un très beau passage de Qu'est-ce que s'orienter dans la pensée? consacré à la liberté d'expression vient également insister sur l'importance de la contribution d'autrui à nos réflexions:

[Penserions]-nous beaucoup, et penserions-nous bien, si nous ne pensions pas pour ainsi dire en commun avec d'autres, qui nous font part de leurs pensées et auxquels nous communiquons les nôtres? Aussi bien, l'on peut dire que cette puissance extérieure qui enlève aux hommes la liberté de communiquer publiquement leurs pensées, leur ôte également la liberté de penser $[\ldots]^{49}$.

Il nous semble cependant nécessaire de préciser que, dans une perspective kantienne, l'apport d'autrui se révèle particulièrement significatif pour tout ce qui touche à des questions d'ordre empirique: croyances, opinions, jugements subjectifs en général, etc. La confrontation de nos propres pensées avec celles d'autrui se révèle alors particulièrement profitable, en ce que nos jugements peuvent en bénéficier. Mais il est bien certain que les principes $a$ priori issus de la raison pure ne peuvent, quant à eux, subir une telle amélioration; si amélioration il y a, elle doit se situer plutôt dans l'usage que nous faisons de ces principes. La question serait donc de savoir si la communication de nos pensées peut rendre notre raison plus apte à cerner et à appliquer des principes a priori. On peut distinguer, à ce niveau, le travail de l'entendement et celui de la faculté de juger: Kant définit le premier comme "le pouvoir des règles» et le second comme le pouvoir de subsumer sous des règles, soit "de distinguer si quelque chose s'inscrit ou non sous une règle donnée (casus datae legis) ». Or, si les règles régissant l'usage de l'entendement peuvent être enseignées et apprises, l'usage de la faculté de juger est, quant à lui, un pouvoir "qui ne se peut nullement apprendre, mais seulement exercer ${ }^{50}$ ». Il faut néanmoins préciser que les jugements étudiés dans la Critique de la raison pure sont des jugements déterminants: à ce titre, la philosophie transcendantale doit être en mesure d'indiquer les cas a priori pour lesquels une règle doit être appliquée - et c'est là précisément le rôle de l'Analytique des principes. Celle-ci doit donc servir de canon pour la faculté de juger en lui enseignant "comment appliquer à des phénomènes les concepts de l'entendement ${ }^{51}$ ", lesquels contiennent la condition de règles $a$ priori. L'exercice de la faculté de juger doit donc, dans le contexte de la Critique de la raison pure, demeurer strictement encadré - ce qui ne va d'ailleurs pas sans rappeler le fait que la liberté de penser doit toujours, pour Kant, s'imposer à elle-même certaines restrictions, contrairement à la liberté de penser sans lois du "génie». Dans ce contexte, la confrontation de nos

49. QO, Ak. VIII, I44.

50. $C R P, \mathrm{~A}_{13} / \mathrm{B}_{1} 72$.

51. CRP, $\mathrm{A}_{3} 32 / \mathrm{B}_{7} 7 \mathrm{I}$. 
propres pensées avec celles d'autrui peut peut-être contribuer, dans une certaine mesure, à développer notre raison — voire à parvenir à une meilleure connaissance des règles régissant notre entendement et à aiguiser notre faculté de juger. Mais il semble moins crucial d'insister sur l'importance de penser en commun en ce qui concerne les principes a priori de la raison ${ }^{52}$.

\section{La nécessaire dimension négative des Lumières}

La nécessaire connaissance de soi qui vient sous-tendre l'entreprise critique gagne ainsi à être comprise dans la perspective offerte par la conception kantienne des Lumières - ce qui permet, par le fait même, de souligner leur contribution à l'usage théorique de la raison. Il vaut la peine de rajouter à cette analyse un aspect supplémentaire: la nécessité de concevoir la réflexivité à l'œuvre dans la Critique comme tâche commandant une perpétuelle rectification de la raison par elle-même. C'est dans cette perspective que nous examinerons finalement d'un peu plus près l'importante dimension négative des Lumières, qui rejoint celle de la Critique de la raison pure en ce qu'elle a pour principale fonction de venir corriger une propension de la raison à l'erreur, causée essentiellement par son penchant $[\text { Trieb }]^{53}$ à élargir ses connaissances de façon illimitée.

Comme le précise la note de Qu'est-ce que s'orienter dans la pensée? citée plus haut ${ }^{54}$, Kant conçoit les Lumières comme «un principe négatif de

52. En s'inspirant de la deuxième maxime du sens commun présentée au $\$ 40$ de la Critique de la faculté de juger, Wood soutient qu'on ne peut développer sa raison qu'en communiquant avec les autres. Il défend ainsi que «La pensée est en soi une activité sociale car elle doit être critique et tester ce qui est pensé à partir d'une pluralité de points de vue [...] Il est possible d'y parvenir au moyen de l'imagination, mais seulement partiellement, et l'imagination doit en tous les cas être régulièrement stimulée par une communication réelle avec ceux dont on essaie d'adopter la perspective. C'est pourquoi Kant dit que l'existence même de la raison dépend de la liberté de communication» (Kant's Ethical Thought, p. 302; ma traduction). Notons que la dernière phrase réfère à un passage de la Critique dans lequel Kant ne tient pas exactement les propos que Wood lui prête, puisqu'il y soutient en fait que l'existence de la raison repose sur la liberté de la critique: "La raison doit, dans toutes ses entreprises, se soumettre à la critique, et elle ne peut par aucun interdit attenter à la liberté de cette dernière sans se nuire à elle-même [...] C'est sur cette liberté que repose même l'existence de la raison [...]» (A738/B766; l'italique est de moi). Si l'existence de la raison dépend bel et bien de sa publicité et, pourrait-on ajouter, de sa communicabilité, il faut tout de même souligner que Kant ne pose pas les échanges (réels) entre êtres raisonnables comme indispensables à cet égard. La communication avec autrui est sans aucun doute d'une grande aide lorsqu'il s'agit de penser par soi-même de façon éclairée (et encore davantage pour exercer sa faculté de juger à poser des jugements réfléchissants), mais sans pour autant constituer une condition de possibilité de l'existence même de la raison.

53. Voir notamment en $C R P, \mathrm{~A}_{5} / \mathrm{B} 8 ; \mathrm{B}_{4} 26 ; \mathrm{A}_{708} / \mathrm{B}_{73} 6$. Soulignons que Kant évoque justement ce "penchant à la connaissance» [Erkenntnistrieb] dans Qu'est-ce que s'orienter dans la pensée? (Ak. VIII, I39n), en ce qu'il est à l'origine du besoin éprouvé par la raison d'aller au-delà des limites de l'expérience.

54. QO, Ak. VIII, I47n. 
l'usage de la faculté de connaître ", ou, comme l'exposera plus tard la Critique de la faculté de juger, d'une "démarche simplement négative ${ }^{55}$ " confiant ainsi un rôle de premier ordre aux Lumières sur le plan spéculatif. Cette qualification négative a retenu l'attention de maints lecteurs de Kant ${ }^{56}$. Certains, comme Monique Castillo, y voient une forme d'inachèvement: les Lumières seraient ainsi "une pratique naïve de la critique dont la portée n'est que négative " et risqueraient conséquemment de stagner à "un stade prépositiviste d'une simple satisfaction cognitiviste, négativement limitée au savoir de l'erreur ${ }^{57}$ ». En clair, les Lumières kantiennes ne seraient rien d'autre qu'une forme de scepticisme. Or, plutôt qu'une marque de naïveté ou de "simple satisfaction cognitiviste ", l'insistance de Kant sur la dimension négative des Lumières nous semble particulièrement justifiée dans le contexte particulier de Qu'est-ce que s'orienter dans la pensée? puisque ce qui est en jeu, dans cet opuscule, est la possibilité de s'orienter dans le "suprasensible» - autrement dit, le rapport de la raison à un savoir qui transgresse les limites de l'expérience et qui constitue pourtant l'objet de prédilection de la métaphysique. Dans ces conditions, les Lumières ne peuvent que coïncider avec le volet négatif du criticisme à l'œuvre dans la Dialectique transcendantale ${ }^{58}$ et donnant lieu, en matière de méthode, à une discipline. Une connaissance adéquate de notre pouvoir de connaître permet en effet de confirmer que le type de savoir en jeu dans cet opuscule, comme, d'ailleurs, dans la Dialectique transcendantale, en est un qui commande la transgression des limites de l'expérience possible; dans ce contexte, les Lumières sous-tendant le criticisme doivent nécessairement avoir une portée négative ${ }^{59}$. Nous reviendrons sur les causes de cette erreur.

Précisons cependant que même hors de ces circonstances particulières, les Lumières gagnent à être comprises comme une démarche essentiellement négative sur le plan formel ${ }^{60}$. D'abord, en ce sens qu'elles ne rajoutent rien à la raison qui n’y soit déjà présent, sinon une meilleure connaissance de son

55. CFJ, Ak. V, 294n.

56. Foucault au premier chef (dans son Qu'est-ce que les Lumières?: "Kant définit 1)Aufklärung d'une façon presque entièrement négative, comme une Ausgang, une "sortie", une "issue" ", p. 564); mais aussi Castillo (Kant: l'invention critique), Deligiorgi (Kant and the Culture of Enlightenment), Allison ("Kant's Conception of Aufklärung»), Fleischacker ( Kant's Enlightenment» dans What is Enlightenment?), etc.

57. Castillo, Kant: l'invention critique, p. I 5-I6.

58. Allison remarque d'ailleurs fort bien ce recoupement entre Lumières et criticisme ( «Kant's Conception of Aufklärung», p. 233).

59. Kant souligne toutefois, dans sa seconde préface, que même ce caractère négatif possède une utilité positive, puisqu'il permet, en limitant la raison spéculative, de faire place à un usage pratique des idées de la raison pure (CRP, BXXIV) — suivant lequel les idées viennent fournir le moyen de mettre des actions en œuvre, plutôt que de chercher à déterminer un objet de l'expérience.

60. Voir à ce sujet Deligiorgi: les Lumières ne concernent pas le contenu, mais bien la forme; il s'agit d'une «manière de penser» (Kant and the Culture of Enlightenment, p. 57). 
propre pouvoir de connaître - les Lumières ne résidant pas dans l'acquisition de connaissances, comme le concevait par exemple Mendelssohn. La note précédemment citée de Qu'est-ce que s'orienter dans la pensée? le montre bien :

[...] Cela suppose bien moins que ne se l'imaginent ceux qui veulent voir l'Aufklärung dans les connaissances acquises; elle consiste plutôt en un principe négatif de l'usage de la faculté de connaitre et il arrive souvent que celui qui est abondamment pourvu de connaissances soit le moins éclairé sur leur usage $^{61}$.

Ce qui ne revient pas à dire que les Lumières soient hostiles au progrès de la connaissance, au contraire; mais elles se situent plutôt au principe de ce progrès, c'est-à-dire dans l'usage même de la raison. Elles sont en ce sens antérieures à toute acquisition de connaissance.

De fait, et c'est là l'autre composante "négative" des Lumières qui rejoint encore une fois l'entreprise critique: elles ont pour tâche de venir rectifier certaines erreurs compromettant l'usage de la raison. Ces errements de la raison pure sont de plusieurs types, évoqués dans la préface à la deuxième édition de la Critique de la raison pure:

C'est alors uniquement grâce à cette Critique que peuvent être entièrement éradiqués le matérialisme, le fatalisme, l'athéisme, l'incroyance des libres penseurs, l'exaltation de l'esprit et la superstition, qui peuvent être universellement dommageables, enfin aussi l'idéalisme et le scepticisme, qui sont plus dangereux pour les écoles et peuvent difficilement passer dans le public ${ }^{62}$.

Ainsi, si Kant caractérise les Lumières de façon essentiellement négative, ce n'est pas parce qu'elles n'ont pas de retombées positives - comme on l'a vu dans la section précédente, chercher la pierre de touche de la vérité en soi se traduit par l'entreprise de connaissance de soi indispensable à la Critique et pouvant certainement être vue comme une réelle avancée -, mais plutôt en raison d'une certaine propension de la raison à l'erreur. Le retour sur soi permettant de corriger cette tendance fait ainsi partie intégrante de l'entreprise critique, tout en s'inscrivant dans la mouvance des Lumières.

Il convient d'abord de remarquer que la superstition et l'exaltation de l'esprit, déjà évoquées précédemment, sont deux types d'erreurs étroitement reliés et auxquels les Lumières kantiennes se proposent expressément de remédier. Dans le contexte de la querelle du panthéisme, l'accusation de Schwärmerei visait tout particulièrement Jacobi et consistait en un rejet de toute législation de la raison sous le prétexte d'une illumination. Ce rejet, loin de résulter en une liberté de penser totale, a au contraire pour effet d'asservir encore plus sûrement la raison - puisque, comme on l'a vu, celleci ne peut demeurer bien longtemps sans lois et se laisse alors guider par

61. QO, Ak. VIII, I47n.

62. CRP, BXXXIV. 
autre chose: en l'occurrence, par des faits empiriques. C'est ainsi que la raison passe de l'exaltation à la superstition, qui est, comme nous l'apprendra la troisième Critique, le préjugé caractéristique d'une raison simplement passive. Ce préjugé consiste à "se représenter la nature comme n'étant pas soumise à des règles que l'entendement, à travers sa propre loi essentielle, lui donne comme fondement ${ }^{63}{ }^{»}$. Pour celui qui est en proie à la superstition, les réalités naturelles peuvent être expliquées non par des lois, mais bien par le recours à de simples faits - ou même par l'intervention de miracles dans la perspective du Conflit des facultés:

La superstition est le penchant à accorder une plus grande confiance à ce qu'on prétend à tort arriver de manière non naturelle plutôt qu'à ce qui se laisse expliquer par des lois de la nature, que ce soit en physique ou en morale ${ }^{64}$.

Contre cette passivité ou hétéronomie de la raison, la Critique de la faculté de juger définira les Lumières comme «libération de la superstition » — en clair, comme la sortie d'un état de minorité intellectuelle, passant ainsi par une connaissance adéquate de notre pouvoir de connaître.

Or, il est intéressant de remarquer que dans la Critique de la raison pure (toutes éditions confondues), Kant ne parle pas de superstition, mais emploie la notion de Schwärmerei en un sens plus large: loin de la limiter au contexte spécifique de la querelle, il l'étend explicitement au domaine de la connaissance et, au final, à tout usage de la raison qui trahirait une méconnaissance de ses lois et des limites de son pouvoir de connaître. De fait, dans un passage presque poétique de l'Analytique des principes, Kant évoque l'image d'un "navigateur exalté » [herumschwärmenden Seefahrer], voguant sur «un océan vaste et agité de tempêtes » autour du pays de l'entendement pur et entraîné «dans des aventures auxquelles il ne peut jamais renoncer, mais qu'il ne peut pourtant jamais non plus conduire à leur terme ${ }^{65}$ ». Parmi les allusions les plus significatives à la Schwärmerei dans la Critique, on peut également rappeler celle portant sur Locke dans le contexte de la Déduction transcendantale. Ainsi, Locke, en dérivant les concepts de l'entendement à partir de l'expérience (ou, pour rejoindre la définition de la superstition: en basant ses explications sur de simples faits), est accusé d'avoir «[ouvert] la porte à l'exaltation de l'esprit [Schwärmerei], parce que la raison, une fois qu'elle a des droits de son côté, ne se laisse plus tenir en lisière par de vagues appels à la modération ${ }^{66}{ }$. Cette association de l'empirisme lockéen à la Schwärmerei n'a de sens que si on comprend cette dernière de façon plus large - en tant que faute commise par la raison à propos de ses propres

63. CFJ, Ak. V, 294.

64. Conflit des facultés, Ak. VII, $65 \mathrm{n}$.

65. CRP, A234-236/B294-295. L'aspiration de la raison à un savoir qui dépasserait les limites de l'expérience est aussi associée à la Schwärmerei en AXIII; B42I ; A8I 9/B847.

66. CRP, $\mathrm{B}_{\mathrm{I}} 28$. 
capacités et des concepts qu'elle utilise. Hume n'a d'ailleurs pas droit au même traitement, puisque Kant, dans le même alinéa de la Déduction transcendantale, précise qu'il a pour sa part su reconnaître que des concepts dérivés empiriquement ne pouvaient permettre d'aller au-delà des limites de l'expérience. Aucune accusation d'exaltation n'est donc portée à son endroit, le scepticisme représentant bien plutôt l'excès inverse - puisqu'il s'empresse de borner l'entendement.

Mais la Critique de la raison pure ne se borne pas à poser un diagnostic: elle vient aussi montrer les causes de ces dérives, dont la connaissance se révèle indispensable. Il s'agit d'erreurs plus fondamentales car se rapportant directement à la faculté de juger, et que toute recherche de la vérité doit prendre en considération.

On a vu que, pour Kant, la vérité consiste en un accord entre la connaissance et son objet. Or, comme c'est par le jugement que l'objet peut être mis en rapport avec notre entendement, c'est aussi en lui qu'il faut chercher la possibilité de l'erreur: "vérité ou apparence sont, non pas dans l'objet en tant qu'il est intuitionné, mais dans le jugement porté sur lui, en tant que cet objet est pensé67 ${ }^{6}$. De fait, l'erreur ne peut se situer que dans le jugement lui-même, et non dans les sens ou dans l'entendement en euxmêmes; la sensibilité se borne à nous donner l'objet et l'entendement, à le penser. La première demeure néanmoins le fondement de l'erreur, puisque c'est par son «influence inaperçue» sur le second que surviennent les jugements erronés. Kant fait allusion pour la première fois à la faillibilité de la faculté de juger dans son introduction à l'Analytique des principes: il y mentionne qu'il appartient à la critique de prévenir les «faux pas» (lapsus judicii) ${ }^{68}$ de cette faculté. Ces faux pas peuvent être de plusieurs types: celui de Locke, évoqué plus haut et ayant donné lieu à une exaltation de l'esprit, en est un qui relève de l'amphibologie des concepts de la réflexion ${ }^{69}$. Cet appendice, situé à la charnière de l'Analytique des principes et de la Dialectique transcendantale, fait état des erreurs découlant de la confusion entre usage empirique et usage transcendantal de l'entendement. La faculté de juger prétend en ce cas se passer du schématisme et se rapporter directement aux choses en soi; l'erreur consiste alors à avoir négligé la dualité des sources de la connaissance. C'est la réflexion transcendantale qui doit permettre d'y remédier — c'est-à-dire, «l'acte par lequel je rapproche la comparaison des représentations en général de la faculté de connaissance où elle trouve sa

67. $C R P, \mathrm{~A}_{293} / \mathrm{B}_{35}$ O.

68. $C R P, \mathrm{~A}_{35} / \mathrm{B}_{7} 74$.

69. Au même titre, d'ailleurs, que celui de Leibniz: «Leibniz intellectualisait les phénomènes, tout comme Locke, à travers son système de noogonie [...], avait globalement sensualisé les concepts de l'entendement» (CRP A27I/B327). 
place, et par lequel je distingue si c'est comme appartenant à l'entendement pur ou à l'intuition sensible qu'elles sont comparées les unes aux autres ${ }^{70}$ ».

Or, ce type d'erreur est presque banal si on le compare à celui qui fait l'objet de la Dialectique transcendantale, lequel trahit plutôt une opacité inhérente à la raison. L'erreur abordée dans la Dialectique concerne des principes dont l'usage ne pourra jamais être appliqué à l'expérience: les principes transcendants de la raison pure. Cette erreur, beaucoup plus tenace, est causée par une illusion persistante: l'apparence transcendantale. Celle-ci consiste en l'illusion d'une extension de l'entendement pur, qui nous incite en fait à repousser les limites de l'expérience. Et, de par la nature de notre raison, l'apparence transcendantale ne peut jamais être complètement dissipée - même une fois mise au jour ${ }^{71}$. Il s'agit d'une illusion "qui ne saurait être évitée, pas plus que nous ne parvenons à éviter que la mer nous paraisse plus élevée au large que près du rivage ${ }^{72}$ ", et qui donne lieu à un malentendu [Missverständnis] de la raison avec elle-même. Mue par son penchant à la connaissance ou, plus exactement, par son penchant à vouloir trop connaître, la raison en vient à se méprendre sur son propre compte, puisqu'elle infère, de ce penchant, la possibilité de faire un usage constitutif des idées de la raison pure. Tout ce à quoi la critique peut aspirer est de nous y rendre plus attentifs et de nous permettre ainsi d'éviter autant que possible ce malentendu de la raison avec elle-même, ce qui est une entreprise au demeurant fort difficile et exigeant un effort constant. L'utilisation transcendante des idées de la raison pure trahit en effet ce que Kant qualifie de "raison paresseuse ", soit une raison qui s'autorise certains débordements parce qu'elle considère sa tâche achevée et préfère s'abandonner au repos - ce qui mène à sa corruption et à sa ruine ${ }^{73}$. Contrairement à ce que Kant dénomme "philosophie expérimentale ${ }^{74}$ ", la philosophie transcendantale ne possède en effet nul correctif qui lui permette de dissiper aisément le malentendu. On peut ainsi comprendre l'erreur faisant l'objet de la Dialectique transcendantale comme symptôme d'un manque de transparence inhérent à la raison, auquel doit impérativement venir remédier la raison critique des Lumières sous peine de graves erreurs - la Schwärmerei de la querelle du panthéisme en est un exemple.

70. $C R P, \mathrm{~A}_{26}$ I/B 317.

71. J.-L. Nancy qualifie judicieusement l'apparence transcendantale de "possibilité pathologique de l'erreur ", expliquant que la critique ne peut empêcher la raison de s'abandonner «à cette pulsion qui la porte à juger hors des limites de l'expérience» («Lapsus judicii »; p. 89). Il suggère par ailleurs, de façon fort intéressante, que la connaissance de ce lapsus nous permet elle-même de parvenir à une meilleure connaissance de nous-mêmes.

72. $C R P, \mathrm{~A}_{297} / \mathrm{B}_{3} 54$.

73. CRP A689-690/B717-7I8.

74. Voir CRP A424-425/B452. Experimentalphilosophie est parfois corrigé par «physique » ou "philosophie de la nature ». 
Ainsi, un problème de taille doit immanquablement venir perturber l'entreprise de connaissance de soi qui fait l'objet des développements de la Critique de la raison pure: celui de l'erreur, sous toutes ses formes. Celle-ci, pour être rectifiée, bénéficie d'ores et déjà des Lumières à l'œuvre dans la Critique. Rappelons que cette dimension négative ne signifie pas que les Lumières à l'œuvre dans la Critique de la raison pure ne constituent pas un apport positif à notre connaissance. Chercher en soi-même la pierre de touche de la vérité de ses jugements se traduit, on l'a vu, par la connaissance de soi qui fait l'objet des développements de la Critique de la raison pure. C'est là en soi, nous semble-t-il, une avancée positive (et une de taille) $)^{75}$, bien qu'elle porte sur le pouvoir de connaître lui-même et non sur ses objets - et, bien sûr, qu'elle ne puisse jamais permettre de procurer de l'extension à l'entendement. Mais la propension de la raison à l'erreur la plus redoutable de toutes, présentée dans la Dialectique transcendantale, implique que la réflexivité à l'œuvre dans la Critique ne puisse jamais être regardée comme une entreprise définitivement achevée. Les Lumières culminent ainsi dans une entreprise permanente de rectification, en raison de ces «erreurs momentanées qui ont sans cesse besoin d'être surmontées » et se traduisant par une « dialectique naturelle et inévitable de la raison pure $^{76}$ » - laquelle n'est pas causée par un manque de connaissances, mais bien par l'illusion décrite plus haut. Si l'erreur peut, à force de persévérance, être surmontée, l'apparence transcendantale elle-même résistera toujours à la connaissance de soi et appelle ainsi une vigilance constante.

\section{Conclusion}

On a vu que, même si Kant n'a publiquement exposé sa conception des Lumières qu'en $\mathrm{I} 784$, certains aspects de cette conception étaient déjà bien présents dans la Critique de la raison pure, et ce, dès sa première édition. L'utilisation même du terme d'Aufklärung faite par Kant à cette époque, bien qu'elle ne corresponde pas exactement à celle qu'il devait en faire à compter de 1784 , suggère que la clarification conceptuelle qu'elle commande doit être comprise comme partie prenante d'une conception de la philosophie privilégiant le fait de penser par soi-même à l'inculcation de doctrines préétablies. Lumières et critique nous semblent ainsi devoir se rejoindre dans leur essence même.

75. Nous suivons ici Zöller («Aufklärung über Aufklärung. Kants Konzeption des selbständigen, öffentlichen und gemeinschaftlichen Gebrauchs der Vernunft», p. 90), qui remarque qu'avec la définition de penser par soi-même proposée par Qu'est-ce que s'orienter dans la pensée? les Lumières perdent la tournure strictement privative qu'elles avaient dans Qu'est-ce que les Lumières? Dans ce dernier opuscule, le fait de penser par soi-même était en effet conçu comme un geste exclusivement privatif, en tant que libération de la tutelle intellectuelle. À partir de l'opuscule de I786, les Lumières sont plutôt définies par le fait de chercher en soi la pierre de touche de la vérité.

76. CRP A298/B354-355. 
Cette association est d'autant plus frappante si l'on considère les précisions que Kant rajoute à sa conception des Lumières dans le cadre de la querelle du panthéisme avec Qu'est-ce que s'orienter dans la pensée? Les Lumières y sont en effet associées au fait de chercher en soi-même la pierre de touche de la vérité, ce qui ne va pas sans évoquer l'entreprise, centrale à la critique, de connaissance de soi - laquelle consiste, on l'a vu, en une mesure adéquate du pouvoir de connaître de la raison. Cette réflexivité à l'œuvre dans la Critique commande par ailleurs une légitimation publique, c'est-à-dire, qui doit pouvoir être constatée par tout être raisonnable. La connaissance de soi sous-tendant la Critique nous semble ainsi intrinsèquement reliée au fait de penser par soi-même, ce qui confère du même coup une importance prépondérante aux lois devant régir cette liberté de penser de la raison spéculative. Une liberté sans lois, comme celle à laquelle aspire le «génie» dans la perspective de la querelle du panthéisme, est pour Kant une voie sans issue, puisqu'elle est vouée à retomber dans la légalité par tous les moyens possibles - passant ainsi de la Schwärmerei à la superstition. Cette importance des lois pour une raison des Lumières souligne non seulement la nécessité de parvenir à une connaissance adéquate de notre pouvoir de connaître, mais également celle de le limiter proprement lorsque c'est nécessaire. Ce dernier aspect ne va pas sans évoquer la dimension négative des Lumières, telle que nous l'avons présentée dans la troisième section. Pour Kant, celles-ci sont appelées à intervenir de façon d'autant plus cruciale lorsque vient le temps de prévenir et de rectifier les dérives de notre faculté de connaître. Nous avons vu que celles-ci sont dues à divers types d'erreurs imputables à notre faculté de juger, la plus redoutable demeurant l'erreur fondamentale de la Dialectique transcendantale, qui consiste à faire un usage transcendant des principes de la raison pure - lesquels ne peuvent jamais être appliqués à une expérience possible. Mais cette erreur n'est pas seulement de taille: elle est également particulièrement tenace, en ce que l'apparence transcendantale qui la fonde ne pourra jamais être surmontée. Le penchant de notre raison à y succomber commande donc une réflexivité qui fait partie intégrante de la connaissance de soi prescrite par la Critique. Car sans elle, c'est la Schwärmerei qui guette la raison, comme le préfigure d'ailleurs un passage de l'Architectonique de la raison pure:

En suivant tout le cours de notre Critique, on se sera suffisamment convaincu que, bien que la métaphysique ne puisse pas être le soubassement de la religion, elle doit pourtant en demeurer toujours comme le rempart, et que la raison humaine, qui est déjà dialectique par l'orientation de sa nature, ne peut jamais se passer d'une telle science, qui vient la brider et qui, grâce à une connaissance scientifique et pleinement éclairante [einleuchtendes] de soi-même, écarte les dévastations que, sinon, une raison spéculative dépourvue de lois susciterait tout à fait immanquablement aussi bien dans la morale que dans la religion ${ }^{77}$. 